\title{
MicroRNA-127 Post-Transcriptionally Downregulates Sept7 and Suppresses Cell Growth in Hepatocellular Carcinoma Cells
}

\author{
Jiansheng Zhou ${ }^{a, c}$ Shan Lu ${ }^{a, c}$ Shengsheng Yang ${ }^{a}$ Huan Chen ${ }^{a}$ Hanping Shib $^{b}$ \\ Mingyong Miao ${ }^{a}$ Binghua Jiao ${ }^{a}$ \\ aDepartment of Biochemistry and Molecular Biology, The Faculty of Basic Medical science, Second \\ Military Medical University, Shanghai, 'bepartment of General Surgery, The First Affiliated Hospital,

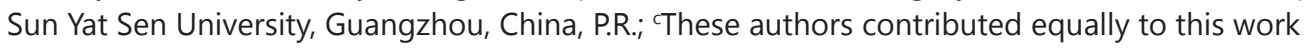

\section{Key Words \\ HCC • MiR-127 • Sept7}

\begin{abstract}
Background/Aims: Hepatocellular carcinoma is one of the most common cancers worldwide. It has been suggested that microRNAs, a class of small regulatory RNAs, are associated with tumorigenesis by targeting the mRNAs of hundreds of genes that modulate a variety of biological processes, including cellular differentiation, apoptosis, metabolism, and proliferation. Methods/Results: we analyzed the expression levels of mir- 127 in $33 \mathrm{HCC}$ and non-cancerous tissues using qRT-PCR. MiR-127 is downregulated in $69.7 \%$ of HCC tissues compared with adjacent normal tissues, but its expression level is not correlated with the TNM stage, AFP level, or age. In vitro, miR-127 can arrest Huh7 at the G2/M phase and inhibit Huh7 cell proliferation. In an in vivo xenograft model, the overexpression of miR-127 can inhibit Huh7 cell tumorigenicity. The luciferase reporter and western blot results confirm that miR127 downregulates Sept7 expression by targeting its 3'UTR. Furthermore, the knockdown of Sept7 has the same effect on cell proliferation as the overexpression of miR-127 in Huh7 cells. Conclusion: miR-127 plays a tumor-suppressor role and can serve as a potential diagnostic biomarker for HCC.
\end{abstract}

Hanping Shi, Mingyong Miao Binghua Jiao
Department of Biochemistry and Molecular Biology, Faculty of Basic Medical science, Second Military Medical University; 800, Xiangyin Road, 200433, Shanghai, (P.R. China) And Department of General Surgery, The First Affiliated Hospital, Sun Yat Sen University, Zhongshan II Road, Guangzhou, 510080, Guangdong, (P.R. China) E-Mail shihp@vip.163.com, E-Mail miaomy@163.com, E-Mail jiaobh@live.cn 


\section{Introduction}

Hepatocellular carcinoma (HCC) is the sixth most common cancer and the third largest cause of cancer mortality worldwide $[1,2]$. Most HCC cases are initially diagnosed at an advanced stage because of the absence of routine screening means. Therefore, the identification of a reliable biomarker for HCC is of great importance. Because the onset and progression of HCC is a multistep process with genetic and epigenetic changes [3, $4]$, the identification of epigenetic changes in miRNAs (miRs) and their target genes may provide effective tools for the early diagnosis and cancer therapy of HCC. MicroRNAs are a class of naturally occurring, small noncoding RNAs that contain $\sim 22$ nucleotides. In general, microRNAs regulate gene expression by targeting the 3 ' untranslated region (3'UTR) of a specific mRNA $[5,6]$. Initially identified in B-cell chronic lymphocytic leukemia [7], the abnormal expression of microRNAs has been reported in many tumors. Similarly to protein-coding genes, microRNAs can act as oncogenes or tumor-suppressor genes [8]. In human HCC, a number of miRNAs, such as miR-7, miR-101, miR-122, miR-125, miR-139, miR-145, and miR-211, have been identified to be aberrantly expressed and can be applied as prognostic markers [9]. The expression level of microRNA-26, which is downregulated in HCC, is associated with survival and response to adjuvant therapy with interferon $\alpha$ [10]. The previous studies all indicate that miRNAs play important roles in HCC.

miR-127 was initially identified by Altuvia et al. through bioinformatic and sequence analysis, and its coding gene is located within the imprinted Dlk1/Gtl2 region, one of the largest microRNA clusters $[11,12]$. miR-127 plays an important role in fetal lung development, and the overexpression of miR-127 in a fetal lung at an early stage can lead to defective terminal bud formation and uneven lung development [13]. In a previous study, we showed that miR-127 can modulate liver regeneration by targeting BCL6 and SETD8 [14]. In vitro, miR-127 can modulate cell proliferation and senescence by targeting BCL6 [15] and regulate cell migration via MMP13 in HCC cells [16]. A recent in vivo study linked mir-127 to HCC carcinogenesis: miR-127 is downregulated after the administration of a methyl-deficient diet, which induces hepatocarcinogenesis in rat [17]. However, the underlying mechanism has not yet been extensively studied.

To explore the involvement and possible mechanism of mir-127 in HCC, we analyzed the expression levels of mir-127 in 33 HCC and non-cancerous tissues. A functional study was subsequently conducted on Huh7 cells using miR-127 mimics. Cell growth and cell cycle distribution analyses, as well as a luciferase reporter assay, were also performed.

\section{Materials and Methods}

\section{Tissue specimens}

We collected 33 paired HCC tissue samples (cancerous and surrounding nontumor hepatic tissues) from patients with HCC during 2007-2011 at Shanghai Oriental Hepatobiliary Hospital. The corresponding non-tumor tissues were obtained at least $5 \mathrm{~cm}$ away from the primary tumor.

\section{Cell culture and transient transfection}

The cell lines LO2 and Huh-7 were purchased from the Type Culture Collection of the Chinese Academy of Sciences (Shanghai, China). All of the cells were cultured in Dulbecco's Modified Eagle's medium (DMEM) (Hyclone, USA) supplemented with $10 \%$ fetal bovine serum (Hyclone, USA) at $37^{\circ} \mathrm{C}$ in $5 \% \mathrm{CO}_{2}$ and subcultured every 2 3 days. miR-127 mimics or a nonspecific sequence (negative control RNA) and Sept7 siRNA and its negative control RNA were obtained from GenePharma (Shanghai, China). The cells were seeded on six-well plates at a density of $3 \times 10^{5}$ cells/well. The transfections were performed using the FuGENE HD Transfection Reagent (Roche, Basel, Switzerland) according to the manufacturer's instructions. The transfected cells were harvested at 36 48 h. 


\begin{tabular}{|c|c|c|}
\hline Cellular F & Cell Physiol Biochem 2014;33:1537-1546 & \\
\hline and Biochemistry & $\begin{array}{l}\text { DOI: 10.1159/000358717 } \\
\text { Published online: May 14, } 2014\end{array}$ & $\begin{array}{l}\text { O } 2014 \text { S. Karger AG, Basel } \\
\text { www.karger.com/cpb }\end{array}$ \\
\hline
\end{tabular}

Isolation of total $m R N A$ and $q R T$ PCR analysis of miRNA expression

The total RNA from the prepared HCC samples and cells was isolated using the TRIzol reagent (Invitrogen), and the cDNA was synthesized following the manufacturer's protocols (MBI Fermentas). The miR-127 sequence was obtained from the miRBase database (http://www.mirbase. org). qRT-PCR was performed using a standard SYBR-green PCR kit (DBI), and the gene-specific PCR amplification was performed using the Applied Biosystems 7300 Sequence Detection system (Applied Biosystems, USA). The qRT-PCR reactions, including the no-template controls, were performed in triplicate. The miR-127 expression was calculated using $\Delta \mathrm{Ct}=\left(\mathrm{Ct}_{\text {miR-127 }}{ }^{-}\right.$ $\mathrm{Ct}_{\mathrm{U} 6}$ ), and the expression levels of the other genes were calculated using $\Delta \mathrm{Ct}=$ $\left(\mathrm{Ct}-\mathrm{Ct}_{\beta \text {-actin }}\right)$. The relative expression of miR-127 was calculated using the $2^{-\Delta \Delta \mathrm{Ct}}$ method $\left(\Delta \Delta \mathrm{Ct}=\Delta \mathrm{Ct}\right.$ tumor $\left.^{-}-\Delta \mathrm{Ct}{ }_{\text {normal }}\right)$. The primers used are listed on Table 1.

\section{Cell cycle analysis by flow cytometry}

The cells were transfected with miR-127 mimics or the negative control. After incubation for $48 \mathrm{~h}$, the cells were harvested. For the cell cycle analysis, the cells were fixed with $75 \%$ etha-
Table 1. Primer used in the qRT-PCR analysis

\begin{tabular}{cl}
\hline Primer Names & Sequences (5'-3') \\
\hline miR-127 & AGAGGCGGACGGTGTCGTAGTTGAAGTGAGCCGTCCGCCTCTAGCCAAGC \\
& GATGTCGGATCCGTCTGAGC \\
& CGGTGTCGTAGTTGAAGTGAG \\
U-6 & GTGCCTGCTTCGGCAGCACATA \\
& TGGAACGCTTCACGAATTTGCGTGTC \\
3-Actin & TGATATCGCCGCGCTCGTCG \\
& ACCCATGCCCACCATCACGC \\
Bcl-6 & GAGCCTTTGCCCCCAGCCTG \\
& GGGGTTGGCCACAGGCATCC \\
Mapk4 & TGGTCGGAAATCTTCGTTTC \\
& ATTGGTGCCTTCAACCTCAC \\
Setd8 & GCAAACTTACGGATTTCTACCCT \\
& CATTCCTTCTTCCTTCCCACTT \\
Sept7 & AGGGCAGCTGACTAAGAGCCC \\
& TCATTTGCTCATGGCGCCGCT \\
\hline
\end{tabular}

Table 2. Vector construction, miR-127 mimics, and Sept 7 siRNA sequences

\begin{tabular}{cl}
\hline NAME & Sequences (5'-3') \\
\hline Sept7 3'-UTR & ACGTCTAGAGACCACCAGTTATGTATTAGTTGC \\
& GATGGCCGGCCGGTAGAAACAGGCTGGTCGTA \\
Sept7-Mu 3'-UTR & CATCAGTGTTTGTTGACGCAGTTTGACCAATTTGC \\
& GCAAATTGGTCAAACTGCGTCAACAAACACTGATG \\
miR-127 mimics & UCGGAUCCGUCUGAGCUUGGCU \\
& CCAAGCUCAGACGGAUCCGAUU \\
miRNA NC sense & UUCUCCGAACGUGUCACGUTT \\
& ACGUGACACGUUCGGAGAATT \\
siRNA NC & UUCUCCGAACGUGUCACGUTT \\
& ACGUGACACGUUCGGAGAATT \\
Sept7-siRNA & UCACCUUCACAAGCAAGUUTT \\
& AACUUGCUUGUGAAGGUGATT \\
\hline
\end{tabular}

nol overnight and then washed twice with ice-cold phosphate buffered saline (PBS) containing 2\% FBS. The cells were then centrifuged, resuspended in RNase-containing (1:100 in dilution) PBS on ice, stained with PI for $30 \mathrm{~min}$, and analyzed using a flow cytometer. The data acquisition and analysis were performed using a FACS cytometer (FACS, CA, USA). A total of $1 \times 10^{5}$ cells were scanned in each analysis. Each experiment was repeated at least three times.

Luciferase reporter constructs, site-directed mutagenesis, and luciferase reporter assay

The 3'-UTR of Sept7, which contains the miR-127 response element, was cloned into the pGL4.13 luciferase reporter vector (Promega) between the XbaI and FseI restriction sites using a directional RT-PCR cloning strategy. The 3'-UTR mutant of Sept7 was synthesized by PCR using the primers listed in Table 2. The resulting luciferase reporter constructs, pGL-Sept7-UTR and pGL-Sept7-Mu-UTR, which contain the wild-type 3'-UTR and the mutant 3'-UTR of Sept7, respectively, were sequenced to ensure accuracy. Huh7 cells were seeded in a 24 -well plate $\left(1 \times 10^{5}\right.$ cells per well $)$ and transfected with $200 \mathrm{ng}$ of the indicated luciferase reporter constructs with miR-127 mimics or miRNA NC. Each sample was cotransfected with $20 \mathrm{ng}$ of the renilla luciferase control vector pGL4.70 (Promega) to monitor the transfection efficiency. The pGL4.13 control vector was used as a control. Forty-eight hours after transfection, all of the protein extracts were analyzed using the dual luciferase reporter assay system (Promega).

\section{Western blot analysis}

The tissues and cells were lysed in RIPA lysis buffer (Beyotime). The lysates were sonicated and centrifuged at $12,000 \mathrm{rpm}$ and $4^{\circ} \mathrm{C}$ for $10 \mathrm{~min}$. The same amounts of protein were separated using $10-15 \%$ 
Fig. 1. miR-127 expression in HCC tissues and HCC cell lines. The miR127 expression levels were determined by qRT-PCR analysis and normalized to the U6 level. The data from three independent experiments are shown as the means \pm SD. (A) miR-127 expression in paired HCC cancerous and adjacent normal tissues. The expression level was calculated by $\Delta \mathrm{Ct}=\left(\mathrm{Ct}_{\text {miR-127 }}-\mathrm{Ct}_{\mathrm{U} 6}\right) \cdot(\mathrm{B})$ Expression level of miR-127 in HCC cell lines normalized to the miR-127 expression level in LO2 cells. (C, D, E, and F) Relative expression of miR127 in cancerous tissues grouped by metastasis, TNM stage, serum AFP concentration $(\mu \mathrm{g} / \mathrm{L})$, and age.

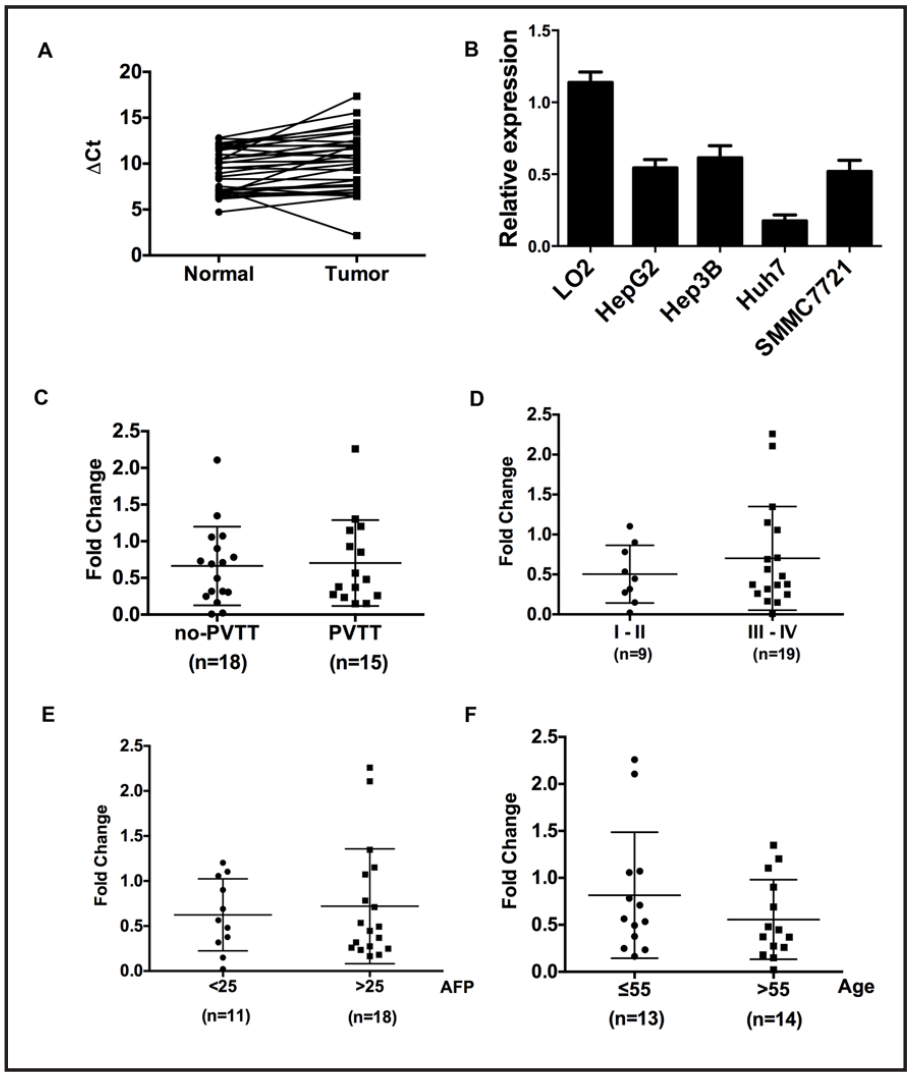

odium dodecyl sulfate-polyacrylamide gel electrophoresis (the exact concentration was determined by the molecular weight of the detected proteins) and transferred to nitrocellulose membranes (Millipore). For immunodetection, the membranes were incubated with specific antibodies (anti-Sept7 and anti- $\beta$-actin; Sigma). The immunoblots were developed using horseradish peroxidase (HRP)-coupled anti-mouse or antirabbit secondary antibodies (ProteinTech Group) and then detected with enhanced chemiluminescence (Pierce Biotechnology). The $\beta$-actin protein was used as a control.

\section{Bioinformatics and statistical analysis}

The predicted target genes of miR-127 were determined by Targetscan (http//www.targetscan.org) and PicTar (http//pictar.mdc-berlin.de/). The results were compared using Student's t test and one-way ANOVA, and the data are expressed as the means and standard deviations of at least three independent experiments. All of the P values were two-tailed and were obtained using GraphPad prism 6. A P value of less than 0.05 was considered statistically significant.

\section{Results}

MiR-127 is downregulated in HCC and hepatoma cell lines

To determine whether miR-127 is decreased in hepatocellular cancer, qRT-PCR was used to analyze 33 paired HCC tissue samples. Using U6 as the endogenous control, the results showed that miR-127 is downregulated in $69.7 \%(23 / 33)$ of cancer tissues compared with the matching adjacent normal tissues, and the expression level of miR-127 in HCC was significantly lower than that observed in the adjacent tissue $(\mathrm{P}<0.01$; Fig. $1 \mathrm{~A})$. In a previous study, we showed that the expression level of miR-127 in Huh7, a HCC cell line, is lower compared with the levels observed in the rat liver, BRL3A cells, and primary hepatocytes [14]. Therefore, the expression level of miR-127 was further examined in the human hepatic cell line LO2 and several hepatoma cell lines, including HepG2, Hep3B, Huh7, and SMMC7721. Our data reveal that a significant reduction of miR-127 expression is observed in liver 


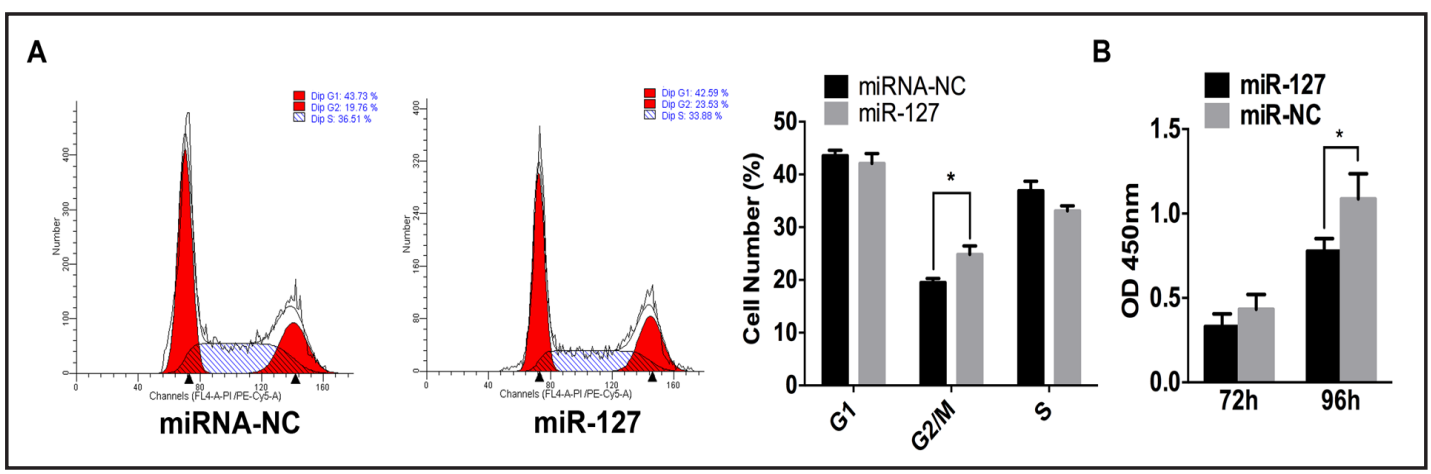

Fig. 2. miR-127 modulates Huh7 cell proliferation. (A) Cell cycle analysis of Huh7 cells transfected with miR-127 mimics (miR-127) or miRNA negative control (miR-NC). The cells were analyzed by flow cytometry. (B) Proliferation of Huh7 cells transfected with miR-127 mimics (miR-127) or miRNA negative control (miR-NC). The proliferation was examined at the indicated times through the methyl thiazol tetrazolium assay.

Fig. 3. MiR-127 suppresses Huh7 cell growth in vivo. (A) In vivo subcutaneous tumor volume growth curves of Huh7 cells transfected with miR-127 mimics (miR-127) or negative control (NC) $(\mathrm{n}=4) .{ }^{*} \mathrm{P}<0.01$ by Student's t-test. (B) Photograph illustrating representative features of mouse tumors derived from Huh7 cells 15 days after treatment with miR-127 or NC. (C) Effects of miR-127 on hepatocellular carcinoma tumor growth in subcutaneous tumor models.

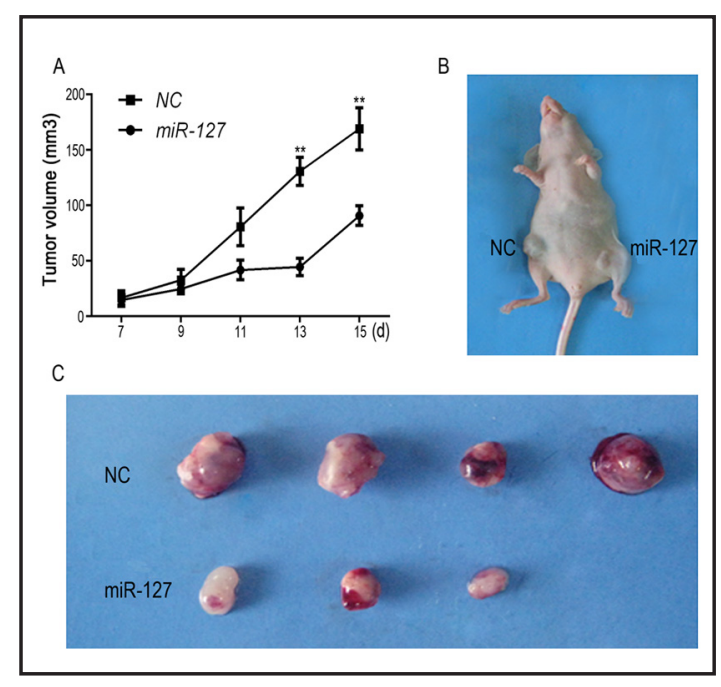

cancer cell lines $(\mathrm{P}<0.01)$ compared with the miR-127 expression level in LO2 cells (Fig. 1B). Collectively, our results indicate that miR-127 is downregulated in HCC tissues and cell lines.

Correlations between the expression of miR-127 and clinicopathological features

The correlation between miR-127 expression and the clinical features of 33 HCC are shown in Fig. 1C-1F. The expression of miR-127 was not significantly associated with portal vein tumor thrombus $(\mathrm{P}=0.8385)$, TNM stage $(\mathrm{P}=0.4044)$, serum AFP concentration $(\mathrm{P}=$ $0.6611)$, and age $(\mathrm{P}=0.2385)$.

miR-127 modulates cell growth and G2/M arrest in Huh7 cells

The frequent downregulation of miR-127 in HCC tissues and cell lines suggests that miR-127 may act as a tumor-suppressor gene in HCC. To confirm this hypothesis, the effect of ectopic miR-127 on cell growth was evaluated in Huh7 cells. Briefly, Huh7 cells were transfected with a miR-127 mimic or specific NC and then subjected to a MTT cell viability assay and flow cytometry analysis. The transfection efficiency of the miR-127 mimic was confirmed by qRT-PCR. As shown in Fig. 2A, the percentages of G2/M-phase cells in miR-127 and miRNA NC groups were $24.837 \pm 1.613 \%$ and $19.497 \pm 0.789 \%$, respectively, and the percentages of cells in the $S$ phase were $33.077 \pm 0.969 \%$ and $36.930 \pm 1.797 \%$, respectively. 
A
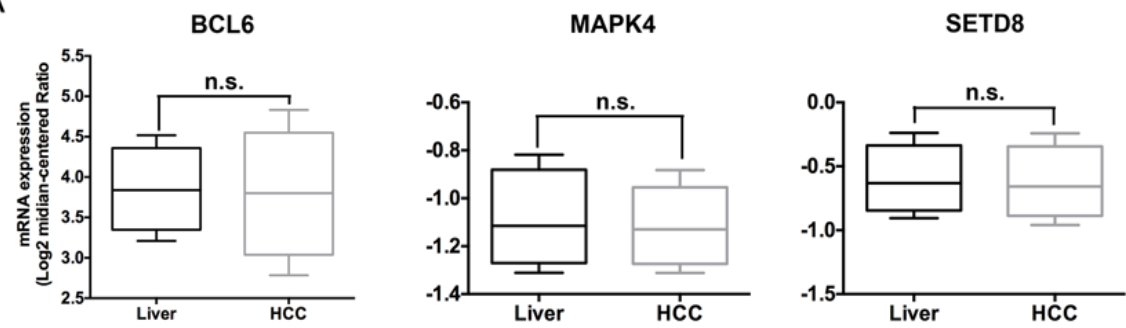

B
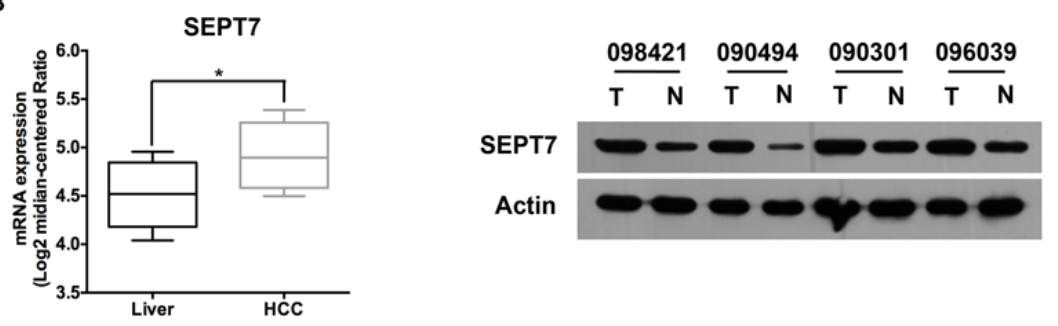

Fig. 4. miR-127 target genes expression in HCC. The mRNA levels of potential target genes of miR-127 were determined using the Oncomine microarray database (https://www.oncomine.org) in normal and HCC tissues. In the box plot, the boxes represent the interquartile range, the whiskers represent the 10th-90th percentile range, and the bars represent the median. (A) The mRNA expression levels of BCL6, MAPK4, and SETD8 exhibit no significant difference between normal liver and HCC. (B) The mRNA expression of Sept7 presents a significant difference between liver and HCC, and western blots for Sept7 in four paired pair tissues were performed.

This finding indicates that Huh7 cells are arrested in the G2/M phase after the transfection of miR-127 mimics. In addition, miR-127 can markedly reduce Huh7 cell growth at $96 \mathrm{~h}(\mathrm{P}$ $<0.05$ ) compared with the NC group, as determined by cell proliferation analysis (Fig. 2 B).

\section{Effect of miR-127 on Huh7 cell tumorigenicity}

To determine the role of miR-127 in hepatocarcinogenesis, an in vivo xenograft model was employed. miR-127-transfected Huh7 cells were injected into one of the posterior flanks of a nude mouse, and NC-transfected Huh7 cells were injected into the opposite posterior flank of the same nude mouse. The growth of tumors in response to the injection of these two cells was monitored based on their volume and weight for a period of approximately two weeks (Fig. 3A). Two weeks after injection, the tumors were removed from the nude mice and photographed. As shown in Fig. 3B-C, the miR-127-transfected cells did not induce tumor formation in one mouse, and the other three tumors formed as a result of the injection of miR-127-transfected cells were significantly smaller than those obtained with the NCtransfected cells $(\mathrm{P}<0.01)$. These data indicate that miR-127 overexpression significantly inhibits the tumorigenicity of Huh7 cells in nude mice xenograft models.

\section{miR-127 downregulates Sept7 expression}

To explore the underlying mechanism of miR-127 in HCC, we sought to identify its target genes. Using bioinformatics tools (TargetScan and miRanda), we selected several potential target genes of miR-127 that may participate in HCC. Although miR-127 is reduced in HCC samples, its target genes, such as Bcl6 [18], Setd8 [14], and Mapk4 [19], which have been confirmed to be important in the onset and progression of cancer expression, are not increased (data not shown). Moreover, the mRNA expression level of these target genes in the Oncomine microarray database (https://www.oncomine.org) shows no significant difference between HCC tissues and normal liver tissues (Fig. 4A) [20]. Interestingly, we found that Sept7 is upregulated at the mRNA and protein levels (Fig. 4B), which indicates 
Fig. 5. Sept7 is a target gene of miR-127 in Huh7 cells. (A) qRT-PCR and western blot analysis for Sept7 after the transient transfection of miR-127. The NC group was used as a control. (B) The repression of the Sept7 gene, which was mediated by its 3'-UTR, was analyzed through a luciferase reporter assay. Huh7 cells were cotransfected with a luciferase reporter vector containing either the 3'-UTR or the mutated 3'-UTR (Mu-UTR) of Sept7 and either miR-127 or NC. The pGL4.13 control vector was used as a control. The wild-type and mutated miR-127 binding sites in the 3'-UTR of this gene are indicated (left). The data from three independent experiments are shown as the means \pm SD.

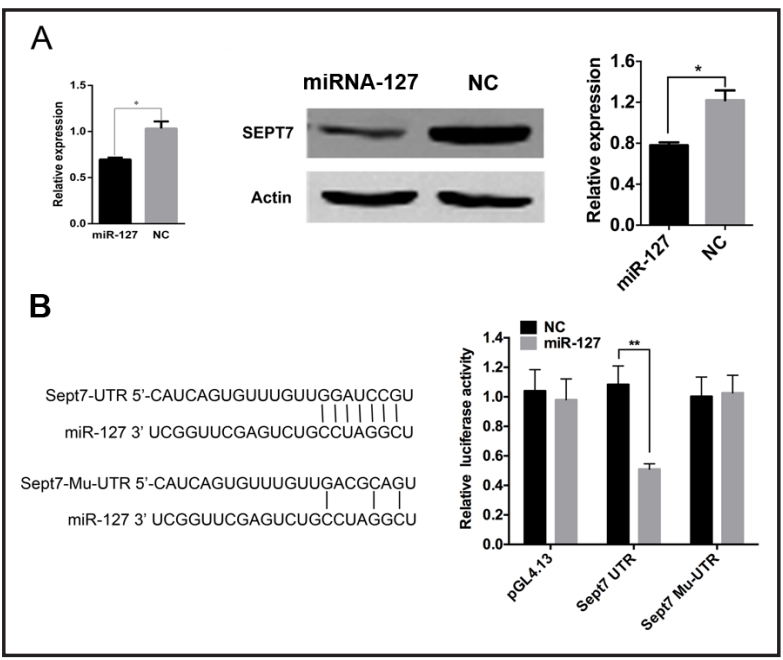

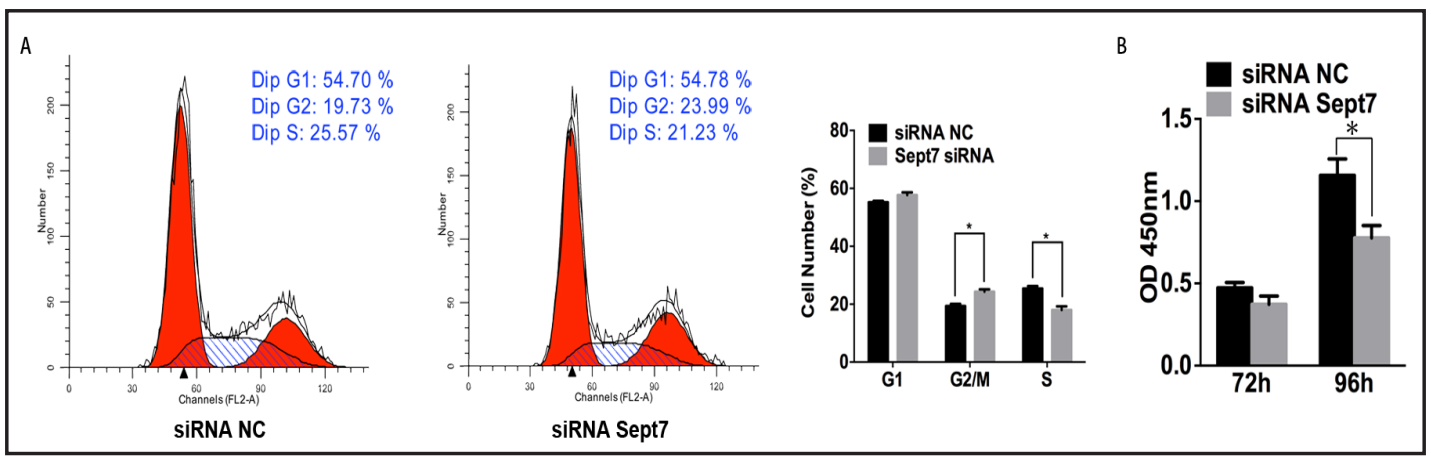

Fig. 6. Downregulation of Sept7 suppress Huh7 cell proliferation. (A) The proliferation of Huh7 cells transfected with Sept7 siRNA or siRNA-NC was examined at the indicated times through the methyl thiazol tetrazolium assay. The data from three independent experiments are shown as the means $\pm \mathrm{SD}\left({ }^{*} \mathrm{P}<0.05\right)$. (B) The proliferation of Huh7 cells transfected with miR-NC or Sept7 siRNA was examined at the indicated times through the methyl thiazol tetrazolium assay.

that its expression is inversely correlated with the expression level of miR-127. This finding is consistent with the data found in the Oncomine microarray database [20].

To validate whether Sept7 is regulated by miR-127, we further examined the expression of Sept7 in Huh7 cells treated with miR-127 mimics. Our data show that miR127 overexpression strongly suppresses Sept7 expression at both the mRNA and protein levels (Fig. 5A). To obtain direct evidence that miR-127 regulates Sept7 expression via posttranscriptional effects on its 3'UTR, we constructed a luciferase reporter plasmid containing the 3'UTR of Sept7. As shown in Fig. 5B, the luciferase activity in the Luc-Sept7-UTRtransfected cells was significantly decreased compared with the luciferase activity of the cells transfected with the Sept7 3'UTR (target site of miR-127) mutant or the NC.

Sept7 suppression is critical for miR-127-induced G2/M arrest in Huh7 cells

To evaluate the effects of Sept7 suppression on HCC progression, we inhibited the endogenous Sept7 expression by specific siRNAs. As shown in the Fig. 6A, the percentages of G2/M-phase cells in the siRNA-NC and Sept7 siRNA groups were $19.360 \pm 0.756 \%$ and $24.303 \pm 0.864 \%$, respectively, and the percentages of S-phase cells were $25.413 \pm 0.816 \%$ and $18.017 \pm 1.244 \%$, respectively. These effects are consistent with those obtained with the overexpression of miR-127. 


\section{Discussion}

The aberrant expression of miRNAs plays an important role in carcinogenesis by affecting multiple target genes [21]. In a clinical retrospective study, a miRNA profile can reflect the developmental lineage and differentiation state of the tumors [22]. In the present study, we found that miR-127 is commonly downregulated in HCC tissues and hepatoma cell lines. Our data are in accordance with some previous studies that showed that miR-127 is downregulated in cancers, including primary prostate tumors, bladder tumors, colon tumors [18], primary breast tumors [15], gastric cancer [19], and glioblastoma [23]. However, in the serum of patients with esophageal squamous cell carcinoma, the concentration of miR-127 is significantly higher than that obtained in normal controls [24].

Although its function is controversial, most lines of evidence support the finding that miR-127 plays a tumor-suppressive role in many tumors. In our investigation, the biological function of miR-127 was also confirmed through in vitro and in vivo studies. Our data show that the overexpression of miR-127 can suppress Huh7 cell proliferation and tumorigenicity.

To further explore the underlying mechanism, we also identified the expression levels of potential target genes of miR-127 in HCC by qRT-PCR and luciferase reporter assays. In gastric cancers, miR-127 can regulate cell migration by targeting Bcl6 and Mapk4 [16, $19]$, and its expression level is associated with the TNM stage of gastric cancer patients [19]. However, our data show that the expression levels of Bcl6 and Mapk4 exhibited no significant changes between HCC tissues and adjacent normal tissues; in addition, we did not find a correlation for miR-127 with portal vein tumor thrombus and TNM stage in HCC. In our study, we found that Sept7 expression is inversely correlated with the expression of miR-127, and a western blot analysis confirmed this finding. Sept7, a member of the septin family, is a highly evolutionarily conserved subfamily of GTPases. Septins can be classified into four groups, namely SEPT2, SEPT3, SEPT6, and SEPT7 $[25,26]$, and all septins influence cellular processes by polymerizing into a hetero-oligomeric protein complex that can form filaments [27]. Moreover, different septins may have different functions. In agreement with this, the knockout of certain septins, such as Sept1, in mice has failed to produce a phenotype, whereas the knockout of Sept7 is embryonic lethal. Sept7 is abundantly expressed in the central nervous system (CNS), and previous studies have shown that its abnormal expression is associated with Alzheimer's disease, nervous system cancer, Down's syndrome, and male infertility [27]. Sept7 is downregulated at the mRNA level in glioma, and the overexpression of Sept7 can suppress glioma cell growth by arresting the cells at the G1/S phase [28, 29]. However, our results show that Sept7 expression is increased in HCC tissues and that the downregulation of Sept7 can inhibit Huh7 cell proliferation by contributing to G2/M blockage, which contradicts its function in glioma. Further studies are needed to uncover the varied mechanisms underlying these biological processes.

Alpha-fetoprotein (AFP) is one of several oncofetal proteins synthesized in large amounts by the fetus, and its synthesis is repressed in healthy adults. Increased AFP levels are often a biomarker for HCC [30,31], but its expression can be very high in some noncancerous liver disease and can be low in some HCC patients [32]. Our results show that miR-127 expression has no correlation with the AFP concentration in the patients' serum (Fig. 1E), indicating that miR-127 may be an independent biomarker for HCC or can be used in combination with AFP or other biomarkers to enhance the accuracy of the diagnosis of HCC.

In summary, our findings demonstrate that miR-127 plays an important role in HCC. The results reveal that miR-127 is decreased in HCC and that the overexpression of miR127 inhibits Huh7 cell growth in vitro and in vivo. Furthermore, we identified that miR-127 can regulate Sept7 expression at the post-transcription level and the Sept7 suppression is essential for miR-127-induced cell cycle arrest. Further studies need to expand the sample size to confirm whether miR-127 can be an effective biomarker for clinical use. 


\section{Acknowledgements}

This work was supported by a National Hi-tech R\&D Program grant (No. 2014AA093513).

\section{Declaration Statement}

The authors have no conflict of interest to disclose.

\section{Reference}

1 Ferlay J, Shin H-R, Bray F, Forman D, Mathers C, Parkin D: Estimates of worldwide burden of cancer in 2008: Globocan 2008. Int J Cancer 2010;127:2893-2917.

-2 Ferenci P, Fried M, Labrecque D, Bruix J, Sherman M, Omata M, Heathcote J, Piratsivuth T, Kew M, Otegbayo J, Zheng S, Sarin S, Hamid S, Modawi S, Fleig W, Fedail S, Thomson A, Khan A, Malfertheiner P, Lau G, Carillo F, Krabshuis J, Le Mair A, World Gastroenterology O: Hepatocellular carcinoma (hcc): A global perspective. J Clin Gastroenterol 2010;44:239-245.

3 Farazi P, DePinho R: Hepatocellular carcinoma pathogenesis: From genes to environment. Nat Rev Cancer 2006;6:674-687.

4 Murakami Y, Yasuda T, Saigo K, Urashima T, Toyoda H, Okanoue T, Shimotohno K: Comprehensive analysis of microrna expression patterns in hepatocellular carcinoma and non-tumorous tissues. Oncogene 2006;25:2537-2545.

Bartel D: Micrornas: Genomics, biogenesis, mechanism, and function. Cell 2004;116:281-297.

Ambros V: The functions of animal micrornas. Nature 2004;431:350-355.

Calin G, Dumitru C, Shimizu M, Bichi R, Zupo S, Noch E, Aldler H, Rattan S, Keating M, Rai K, Rassenti L, Kipps T, Negrini M, Bullrich F, Croce C: Frequent deletions and down-regulation of micro- rna genes mir15 and mir16 at 13q14 in chronic lymphocytic leukemia. Proc Natl Acad Sci U S A 2002;99:15524-15529. Calin GA, Croce CM: Microrna signatures in human cancers. Nat Rev Cancer 2006;6:857-866. Khare S, Zhang Q, Ibdah JA: Epigenetics of hepatocellular carcinoma: Role of microrna. World J Gastroenterol 2013;19:5439-5445.

10 Ji J, Shi J, Budhu A, Yu Z, Forgues M, Roessler S, Ambs S, Chen Y, Meltzer PS, Croce CM, Qin L-X, Man K, Lo CM, Lee J, Ng IO, Fan J, Tang Z-Y, Sun H-C, Wang X-W: Microrna expression, survival, and response to interferon in liver cancer. N Engl J Med 2009;361:437-447.

11 Altuvia Y, Landgraf P, Lithwick G, Elefant N, Pfeffer S, Aravin A, Brownstein M, Tuschl T, Margalit H: Clustering and conservation patterns of human micrornas. Nucleic Acids Res 2005;33:2697-2706.

$\$ 12$ Kircher M, Bock C, Paulsen M: Structural conservation versus functional divergence of maternally expressed micrornas in the dlk1/gtl2 imprinting region. BMC Genomics 2008;9:346.

13 Bhaskaran M, Wang Y, Zhang H, Weng T, Baviskar P, Guo Y, Gou D, Liu L: Microrna-127 modulates fetal lung development. Physiol Genomics 2009;37:268-278.

14 Pan C, Chen H, Wang L, Yang S, Fu H, Zheng Y, Miao M, Jiao B: Down-regulation of mir-127 facilitates hepatocyte proliferation during rat liver regeneration. PloS one DOI: 10.1371/journal.pone.0039151.

15 Chen J, Wang M, Guo M, Xie Y, Cong Y-S: Mir-127 regulates cell proliferation and senescence by targeting bcl6. PloS one DOI: 10.1371/journal.pone.0080266.

16 Yang Z-H, Zhang Y, Wang L: A feedback inhibition between mirna-127 and tgfb/c-jun cascade in hcc cell migration via mmp13. PloS one DOI: 10.1371/journal.pone.0065256.

17 Tryndyak V, Ross S, Beland F, Pogribny I: Down-regulation of the micrornas mir-34a, mir-127, and mir-200b in rat liver during hepatocarcinogenesis induced by a methyl-deficient diet. Mol Carcinog 2009;48:479-487.

18 Saito Y, Liang G, Egger G, Friedman J, Chuang J, Coetzee G, Jones P: Specific activation of microrna-127 with downregulation of the proto-oncogene bcl6 by chromatin-modifying drugs in human cancer cells. Cancer cell 2006;9:435-443.

19 Guo LH, Li H, Wang F, Yu J, He J-S: The tumor suppressor roles of mir-433 and mir-127 in gastric cancer. Int J Mol Sci 2013;14:14171-14184. 
20 Roessler S, Jia H-L, Budhu A, Forgues M, Ye Q-H, Lee JS, Thorgeirsson S, Sun Z, Tang Z-Y, Qin L-X, Wang X: A unique metastasis gene signature enables prediction of tumor relapse in early-stage hepatocellular carcinoma patients. Cancer Res 2010;70:10202-10212.

-21 Meltzer P: Cancer genomics: Small rnas with big impacts. Nature 2005;435:745-746.

-22 Lu J, Getz G, Miska E, Alvarez-Saavedra E, Lamb J, Peck D, Sweet-Cordero A, Ebert B, Mak R, Ferrando A, Downing J, Jacks T, Horvitz H, Golub T: Microrna expression profiles classify human cancers. Nature 2005;435:834-838.

23 Jiang H, Jin C, Liu J, Hua D, Zhou F, Lou X, Zhao N, Lan Q Huang Q, Yoon JG, Zheng S, Lin B: Next generation sequencing analysis of mirnas: Mir-127-3p inhibits glioblastoma proliferation and activates tgf- $\beta$ signaling by targeting ski. OMICS 2014;18:196-206.

-24 Zhang C, Wang C, Chen X, Yang C, Li K, Wang J, Dai J, Hu Z, Zhou X, Chen L, Zhang Y, Li Y, Qiu H, Xing J, Liang Z, Ren B, Yang C, Zen K, Zhang C-Y: Expression profile of micrornas in serum: A fingerprint for esophageal squamous cell carcinoma. Clin Chem 2010;56:1871-1879

25 Kartmann B, Roth D: Novel roles for mammalian septins: From vesicle trafficking to oncogenesis. J Cell Sci 2001;114:839-844.

26 Kinoshita M: Assembly of mammalian septins. J Biochem 2003;134:491-496.

27 Mostowy S, Cossart P: Septins: The fourth component of the cytoskeleton. Nat Rev Mol Cell Biol 2012;13:183-194.

28 Jia Z-F, Huang Q, Kang C-S, Yang W-D, Wang G-X, Yu S-Z, Jiang H, Pu P-Y: Overexpression of septin 7 suppresses glioma cell growth. J Neurooncol 2010;98:329-340.

29 Jia Z, Wang K, Wang G, Zhang A, Pu P: Mir-30a-5p antisense oligonucleotide suppresses glioma cell growth by targeting sept7. PloS one DOI: 10.1371/journal.pone.0055008.

-30 Ball D, Rose E, Alpert E: Alpha-fetoprotein levels in normal adults. Am J Med Sci 1992;303:157-159.

-31 Blumberg B, Larouzé B, London W, Werner B, Hesser J, Millman I, Saimot G, Payet M: The relation of infection with the hepatitis b agent to primary hepatic carcinoma. Am J Pathol 1975;81:669-682.

32 Tateishi R, Yoshida H, Matsuyama Y, Mine N, Kondo Y, Omata M: Diagnostic accuracy of tumor markers for hepatocellular carcinoma: A systematic review. Hepatol Int 2008;2:17-30. 